of symptoms, and the late onset of the vesicular rash. As a result of our experience in this case we recommend that varicella zoster antibodies should be measured in all patients receiving methotrexate and, if the result is negative, patients should be advised to seek medical attention when there is contact with chickenpox so that passive immunisation with varicella zoster immunoglobulins can be given.

Active immunisation with varicella vaccine is available at present in the UK on a named patient basis. Trials with the vaccine have been performed in healthy adults and in healthy children and those suffering from leukaemia. More than $95 \%$ of healthy children seroconvert following vaccination, and more than $90 \%$ are fully protected on subsequent exposure to the virus. In children with leukaemia two doses were required to induce seroconversion in $90 \%$, with a protection rate of $85 \%$. In healthy adults seroconversion was harder to achieve and there was a lower protection rate of $70 \% .^{67}$ Pro- tection of immunosuppressed adults may therefore be harder to achieve and, at present, active vaccination cannot be recommended. Increased awareness, prevention of exposure, passive immunisation, and antiviral therapy are all required in the management of this complication of immunosuppression.

1 Pollock JL, Golding J. Social epidemiology of chickenpox in two British national cohorts. $\mathcal{F}$ Epidemiol Community Health 1993;47:274-81.

2 Committee on Safety of Medicines. Severe chickenpox associated with systemic corticosteroids. Curr Probl Pharmacovigilance 1994;20:1-2.

3 Sherman RA, Silva J Jr, Gandour R. Fatal varicella in an adult: case report and review of the gastrointestinal complications of chickenpox. Rev Infect Dis 1991;13:424-7.

4 Joint Committee on Vaccination and Immunisation. Immunisation in infectious disease. London: HMSO, 1992.

5 Lantner R, Rockoff JB, Demasi J, Ragotzy RB, Middleton Jr E. Fatal varicella in a corticosteroid dependent asthmatic receiving troleandomycin. Allergy Proc 1990;11:83-7.

6 Kuter BJ, Weibel RE, Guess HA, Matthews H, Morton DH, Neff BJ, et al. Oka/Merck varicella vaccine in healthy children: final report of a 2 year efficacy study and 7 year follow up study. Vaccine 1991;September:643-7.

7 Takahashi $M$. Current status and prospects of live varicella vaccine. Vaccine 1992;October:1007-14.

\section{Commentary: severe varicella/zoster and adenovirus infections in immunocompromised patients}

\author{
W R C Weir
}

Both chickenpox and shingles have reputations for being relatively mild. One tends to be regarded as a routine childhood infection, the other as a nuisance - sometimes considerable - in old age. Adenoviruses affecting the immunocompetent host likewise have a reasonably benign clinical repertoire. The advent of powerful immunosuppressive therapies and HIV infections, together with an aging population, has altered this benign scenario. Nonetheless, death from infectious disease - even in immunocompromised patients - should always be regarded as preventable.

In the April issue of Thorax (pages 422-3) Gatnash and Connolly described the case of a 49 year old asthmatic man, immunocompromised by oral corticosteroids and weekly doses of methotrexate, who acquired chickenpox from his son. The son's rash had appeared three weeks before his father was admitted with chickenpox pneumonia which caused his death. The father's rash had developed five days before his admission and treatment with intravenous acyclovir was started on the day of admission. Ideally, he should have had serological testing for herpes virus varicella/zoster at the time his son's chickenpox first appeared. A negative result would have prompted a dose of zoster immune globulin (ZIG), an effective means of preventing chickenpox. ${ }^{1}$ The more vexed question is whether, once the father's chickenpox had developed, earlier admission would have made any difference. A five day delay before starting antiviral therapy might suggest this but, amongst the immunocompromised, there has been no change in the proportion of patients dying with chickenpox since the introduction of acyclovir. ${ }^{1}$ Conversely, previously healthy adults (including pregnant women) appear to benefit from acyclovir when they develop chickenpox pneumonia. ${ }^{2}$ It is therefore important to remind all immunocompromised patients of the potential dangers of chickenpox, particularly if they have not previously had it. A clinical history of chickenpox is not necessarily reliable, and testing of all such patients to identify the seronegative cases at risk might also be wise, albeit expensive.

The patient reported by Morice and Lai in this issue (pages 1221-2) was similar to the one discussed above in that his daughter developed chickenpox five weeks before he presented with chickenpox pneumonia. Nonetheless, it was thought that he had had chickenpox as a child and was presumed to be immune. This therefore underlines the necessity for serological testing of patients at risk, regardless of clinical history. The possibility of second true attacks of chickenpox has been raised by some, ${ }^{3}$ although these may merely be episodes of disseminated skin involvement in patients with herpes zoster.

Adenovirus infection in immunocompetent hosts is, on the whole, harmless. Nonetheless,

\footnotetext{
Coppetts

Muswell Hill, London N10 1JN, 
it has a less than benign repertoire in immunocompromised patients, including severe pneumonitis and haemorrhagic cystitis in bone marrow and renal transplant patients, hepatitis in liver transplant patients, and colitis, hepatitis, and meningoencephalitis in patients with HIV infection. The mortality from adenoviral pneumonitis in immunocompromised patients is approximately $60 \%{ }^{4}$ Sabroe et al on pages 1219-20 describe a 67 year old woman with mixed essential cryoglobulinaemia, treated with prednisolone and cyclophosphamide, who developed adenovirus pneumonitis. Initially the chest radiograph was normal but later developed extensive alveolar shadowing. Tissue culture of material obtained by bronchoalveolar lavage revealed adenovirus. Treatment was commenced with intravenous immunoglobulin and ribavirin and she required mechanical ventilation. She recovered, but whether this was spontaneous or attributable to the treatment given is uncertain. The speed and timing of her recovery, related to the commencement of treatment, suggest that it did, however, play a part.

1 Miller E, Marshall R, Burdien J. Epidemiology, outcome and control of varicella zoster infection. Rev Med Microbiol $1993 ; 4: 222-30$.

2 Haak DA, Zakowski PC, Haak DL, Bryson YJ. Early treatment with acyclovir for varicella pneumonia in otherwise healthy adults: retrospective controlled study and review. Rev Infect Dis 1990;12:788-98.

3 Junker AK, Angus E, Thomas EE. Recurrent varicella-zoster virus infections in apparently immunocompetent children. f Pediatr Infect Dis 1991;10:569-75.

4 Hierholzer JC. Adenoviruses in the immunocompromised host. Clin Microbiol Rev 1992;5:262-74.

\section{Tuberculosis of the sternum presenting as metastatic disease}

\author{
Eithne M T Mulloy
}

\begin{abstract}
The case is presented of a 59 year old Saudi Arabian woman with sternal and vertebral tuberculous osteomyelitis and a benign breast mass simulating metastatic disease. This case illustrates the diagnostic difficulties that may be encountered in the diagnosis of tuberculous osteomyelitis. (Thorax 1995;50:1223-1224)
\end{abstract}

Keywords: tuberculous osteomyelitis, sternum, metastatic disease.

\section{Department of Internal Medicine, Armed Forces Hospital, PO Box 100, Tabuk, Saudi Arabia and Royal College of Surgeons in Ireland E M T Mulloy}

Reprint requests to: DrE M T Mulloy, Department of Respiratory Medicine Beaumont Hospital, PO Box 1297, Pox 129 Dublin 9 Ireland.

Received 5 July 1994 Returned to authors 21 September 1994 Revised version received 24 October 1994 Accepted for publication Accepted for publica
A 59 year old Saudi Arabian woman presented for investigation of a paroxysmal cough of one year's duration. She had no other complaints apart from slight tiredness; in particular, she denied fever, back pain, weight loss, or history of tuberculosis. On examination a $6 \mathrm{~cm} \times 4 \mathrm{~cm}$ mass was palpable over the upper sternum which she stated had been gradually enlarging for three months. The mass was firm and slightly tender with a lobulated surface, but it did not transilluminate. It appeared to be fixed to the sternum, but was not attached to the overlying skin which was of normal colour and temperature. Apart from bilateral expiratory rhonchi, there were no other abnormalities on physical examination. Malignancy of the sternum was suspected.
Radiographic examination showed increased bone density at the junction of the first and second sternal segments on oblique views. The chest radiograph was normal apart from cardiomegaly. Laboratory investigations were unremarkable apart from an erythrocyte sedimentation rate of $50 \mathrm{~mm}$ in the first hour. Serum alkaline phosphatase levels were normal. A needle biopsy specimen of the chest wall mass showed a non-specific fibroblastic proliferative reaction with a dense round cell inflammatory infiltrate at one end of the core of tissue. An MRI scan of the thorax (figure) showed an irregular mass in the upper sternum, expanding the sternal bone, invading the subcutaneous tissues anteriorly and the mediastinum fat posteriorly, and in close contact with the great vessels of the heart. This mass was of low signal intensity on $\mathrm{T} 1$ and high signal intensity on

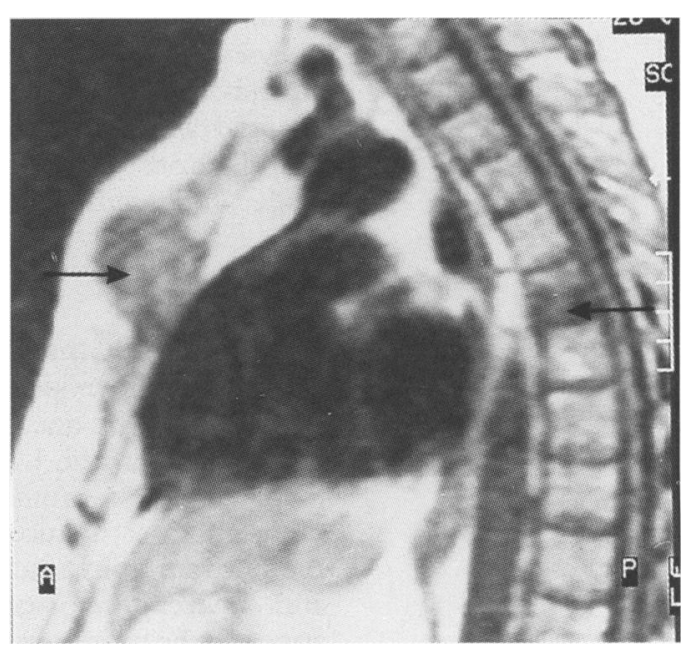

Sagittal MRI scan of the thorax showing the upper sternal mass (anterior arrow) invading the subcutaneous tissue anteriorly and the mediastinum fat posteriorly. A low intensity lesion is also visible in the body of the sixth dorsal vertebra (posterior arrow). The breast mass was visible only on the transverse scan. 